\title{
LAS REVISTAS LITERARIAS. UNA APROXIMACIÓN SISTÉMICA
}

\section{THE LITERARY JOURNAL. A SYSTEMIC APPROXIMATION}

\author{
Ángel Luis SOBRINO VEGAS \\ IES Camilo José Cela, Pozuelo de Alarcón (Madrid) \\ angel.sobrino@educa.madrid.org
}

Resumen: En este artículo se propone una caracterización de la revista literaria desde una perspectiva sistémica, se reflexiona sobre su interés para la historiografía literaria y se plantean unas líneas metodológicas para su descripción y análisis.

Abstract: This paper proposes a characterization of the literary journal from a systemic perspective. There is a reflection on its interests to literary historiography as well as the presentation of some methodological guidelines for its description and further analysis.

Palabras clave: Revista literaria. Hemerografía. Historiografía literaria.

Key words: Literary journal. Hemerographic studies. Literary historiography.

En el marco de la historiografía de nuestra literatura del pasado siglo, se suceden desde hace tiempo los trabajos académicos que se proponen superar, incorporando el resultado de la investigación en archivos personales y 
fondos hemerográficos entre otras fuentes documentales, las limitaciones e insuficiencias de una historia edificada inicialmente sobre el libro como soporte material casi exclusivo de la comunicación literaria. Abundan las declaraciones de estudiosos, críticos e investigadores sobre el interés y la importancia de las revistas literarias (Gracia, 2004: 17; Díez de Revenga, 2005: 11; Ramos Ortega, 2005: 175), se han editado numerosos facsímiles y no cesa la publicación de artículos y trabajos académicos sobre ellas. En muchos casos se observa, sin embargo, que el interés por la revista literaria se debe a que - en la consideración del crítico, del estudioso o del investigador-fueron en su momento un laboratorio o vivero de obras y figuras, transformado luego con el paso del tiempo en yacimiento de materiales que deben ser decantados por el historiador (Ródenas de Moya, 2004: 19). Se desvirtúa de este modo la naturaleza de la revista literaria, cuya importancia, además, queda reducida al cumplimiento de una función ancilar para lo que tradicionalmente se ha tenido por objetivo principal en este ámbito académico: la obra literaria en su versión definitiva, su génesis a partir de variantes y tentativas, su interpretación.

\section{LOS MEDIOS DE COMUNICACIÓN SOCIAL EN LOS SISTEMAS LITERARIOS}

Cuando se parte de la evidencia de que la significación y la literariedad no son propiedades inherentes de los textos, sino resultado de las actividades cognitivas que los individuos realizan en un determinado contexto de actuación y atendiendo a ciertas convenciones sociales (Schmidt, 1995a: 103), el foco de la actividad investigadora se sitúa, por el contrario, sobre el sistema literario y asume como objetivo principal la descripción y, si es posible, explicación de las acciones, procesos y fenómenos que se desarrollan y producen en su seno. Esto no implica, sin embargo, que las obras literarias y su interpretación queden relegadas, sino que todos los aspectos semánticos y estéticos se deben poner en relación con los agentes y con sus contextos de actuación.

Desde una perspectiva sistémica ${ }^{1}$, la literatura es concebida como un ámbito de actuación social en el que se producen y desarrollan actividades, fenómenos

1 En los inicios del siglo XXI, son bien conocidos los vínculos existentes entre la noción de sistema de Niklas Luhmann, la Teoría de los Polisistemas iniciada por Even-Zohar, la Semiótica de la Cultura de Lotman, los estudios sobre el campo literario de Bourdieu y la Teoría Empírica de la Literatura de S. J. Schmidt (Iglesias Santos, 1999: 9-10). Utilizamos para explicar sucintamente la complejidad del 
y procesos de naturaleza muy variada: desde las acciones de producción, mediación, recepción y transformación (Schmidt, 1991: 429-441; Chico Rico, 1995: 23-24) que realizan en los procesos de comunicación literaria los autores, receptores, editores, libreros, intérpretes, críticos, instituciones educativas, traductores, etc., hasta manifestaciones y fenómenos comunicativos como los procesos de institucionalización y canonización, las interpretaciones normativas, la interferencia literaria y cultural o la intertextualidad cultural (Iglesias Santos, 1994: 331-335; Maldonado Alemán, 2006: 27). Estos elementos y fenómenos heterogéneos, que son interdependientes y configuran un sistema social, se vinculan entre sí por su relación directa o indirecta con unos textos considerados literarios según el concepto de literatura integrado en el sistema de presuposiciones bajo el que los sujetos actúan en los procesos comunicativos (Schmidt, 1991: 282-283). Todo un conjunto de instituciones oficiales, como las que regulan e impulsan el desarrollo de actividades de carácter cultural o las instituciones responsables del sistema educativo (tanto de la regulación de su estructura como de la determinación de los programas educativos o del desarrollo de las actividades de enseñanza); entidades públicas y privadas, como academias, ateneos, fundaciones, círculos culturales y literarios; organizaciones empresariales dedicadas a la producción editorial o grupos sociales menos estables y definidos que los anteriores, como cenáculos, tertulias, grupos literarios, en concurrencia solidaria y/o competencia por el dominio del sistema, están implicados en el mantenimiento de la literatura como ámbito de actuación social. Conforman lo que Itamar Even-Zohar (1999: 49) denomina institución. Mediante su actividad - pública o privada; regulada por el sistema social, caso de las actividades de enseñanza, formalizada por los usos sociales, como conferencias, convocatoria de premios, actos públicos, o de carácter informal, caso de las tertulias, reuniones de grupos literarios, etc.- - estas instituciones, entidades y grupos sociales, que tienen entre sus funciones preservar un repertorio canonizado para que pueda ser transmitido a sucesivas generaciones, establecen como dominante un concepto de literatura; regulan las normas y convenciones que operan en el sistema; proponen modelos y orientaciones estéticas para la producción y recepción de las obras; sancionan las producciones con el rechazo, la legitimación o la canonización; estimulan y apoyan la creación de obras literarias con su publicación, la remuneración por ello, la convocatoria y concesión de premios, su divulgación en otras publicaciones, exposiciones y ciclos de conferencias, la recepción crítica, etc., todo lo cual queda incorporado en el dominio cognitivo

sistema literario términos y conceptos tomados de varias de estas teorías sistémicas con el propósito de ofrecer un modelo claro, no limitado y libre de contradicciones. 
de los individuos durante su proceso de socialización y condiciona su participación en el sistema ${ }^{2}$.

Los medios de comunicación social, bajo la propiedad o el control de estas instituciones, entidades y grupos sociales, articulan la esfera pública de su actividad en tanto que constituyen el resultado de la misma como grupo organizado o son empleados para darla a conocer. Por este motivo, para describir y explicar las condiciones de actuación de los individuos y grupos sociales en los sistemas literarios, resulta imprescindible, por una parte, que se conozca y analice el sistema de medios de comunicación de que se dispone en ellos, sus interrelaciones y la posición de cada medio en el sistema; de otra, que se identifiquen las instituciones, entidades y grupos sociales vinculados a cada medio de comunicación, así como los valores, necesidades, intereses y objetivos que determinan su actividad (Schmidt, 1995b: 267-269).

\section{LAS REVISTAS LITERARIAS}

La publicación de revistas literarias ha sido desde los inicios del siglo XX una de las actuaciones preferentes de los grupos literarios en la esfera pública. No cabe duda de que componen, entre los medios de comunicación social, un conjunto con rasgos y funciones específicos que lo distinguen de otros conjuntos integrados en el sistema. Sin embargo, el propósito de fijar una definición de validez universal para el concepto revista literaria, topa enseguida, como ya advirtiera Rafael Osuna (2004: 19), con su imposibilidad. La idea que un lector cultivado tiene hoy de lo que es una revista literaria no se corresponde con muchas publicaciones que han tenido tal denominación en otros momentos de la historia ${ }^{3}$.

2 El concepto de historia o proceso de socialización es entendido como el proceso por el que un individuo se incorpora a un grupo social, aprendiendo y aceptando las normas sociales válidas en ese grupo, las capacidades y habilidades necesarias para el cumplimiento de esas normas y expectativas, así como los valores, convicciones, etc. pertenecientes a la cultura del grupo. El sistema de condiciones desarrolladas a lo largo de la historia de socialización del individuo es lo que S. J. Schmidt denomina sistema de presuposiciones (1991: 281-283).

3 Por ejemplo, en el Anuario administrativo y estadístico de la provincia de Madrid para el año 1868, de Francisco Javier de Bona, impreso en la madrileña Oficina Tipográfica del Hospicio aquel mismo año, se mencionan, entre los periódicos que se publicaban en Madrid el 1 de julio de 1867, treinta de carácter literario, cifra elevadísima bajo cualquier consideración desde nuestra perspectiva actual. Si nos adentráramos en esta relación y examináramos los títulos que la integran, encontraríamos revistas que hoy consideraríamos de información general, de entretenimiento familiar, de humor o dirigidas explícitamente a un público femenino, como El Año, El Álbum de las Familias, El Ángel del Hogar, Los Dos Hemisferios, La Locura, El Universal, entre otras publicaciones. 
Se puede decir, no obstante, que las revistas literarias vinculadas a una concepción del arte y la literatura, que publicaron textos literarios y cuyas páginas sirvieron a los escritores noveles para darse a conocer surgieron en nuestra literatura en los inicios del movimiento romántico, con publicaciones como El Europeo (Barcelona, 1823) y el Diario Literario y Mercantil (Madrid, 1825), y adquirieron durante las primeras décadas del pasado siglo su moderna configuración. Para Rafael Osuna, su actual fisonomía es el resultado de una historia de especialización. Las revistas literarias, escribe, «han tendido a lo largo de su historia a abandonar la miscelaneidad informativa no estrictamente creadora y a concentrarse en la pura creación, llegando a la especialización en cualquiera de los géneros, sean estos los tradicionales o los hemerográficos» (2004: 21). En efecto, el desarrollo de los medios de comunicación social, con el aumento de la producción y de la difusión entre un público creciente, supone, tanto para el mantenimiento de las publicaciones existentes como para las de nueva creación, la competencia por asumir las funciones que otras publicaciones ya desempeñan, la orientación de la línea editorial en busca de un determinado público o la asunción de funciones que ninguna otra publicación asume; esto es, la articulación de un sólido proyecto dispuesto a competir en el mercado o la especialización, ya sea de los contenidos, ya sea de la línea editorial. Ahora bien, admitida esta afirmación como tendencia general seguida en la evolución del sistema, podríamos caer en el error de considerar que la diversidad que hace imposible una definición de validez universal para el concepto revista literaria se observa sólo en la dimensión diacrónica del sistema.

Si desplegáramos el conjunto de las revistas literarias en su secuencia histórica, observaríamos que tanto el número de las series vivas en cada momento como la diversidad de las publicaciones que responden a tal denominación pasan por periodos de expansión y retracción. La producción hemerográfica está fuertemente condicionada por factores externos. La situación económica, el marco político y legal, el control que los poderes públicos ejercen sobre los medios de comunicación, con el establecimiento de la censura, por ejemplo, son determinantes para la configuración del sistema de medios de comunicación social. En el caso de las revistas literarias, además, la diversidad de su producción mantiene relación de proporcionalidad directa con la intensidad y complejidad de la actividad literaria y cultural. En un sistema literario dominado por una fuerte institución central y en el que opera un único concepto de literatura la producción tiende a la uniformidad, mientras que la diversidad es propia de sistemas complejos en que conviven y compiten diferentes conceptos de literatura. La producción de la 
denominada edad de oro de las revistas literarias españolas y la del periodo republicano, que se caracterizaron, respectivamente, por la uniformidad y la diversidad, nos permite señalar cuanto decimos.

Por otra parte, hay periodos históricos en que es posible observar cómo bajo la denominación revista literaria han coexistido publicaciones de muy diverso carácter. A principios del pasado siglo, por ejemplo, en relación con el fenómeno de las colecciones editoriales que se inicia en 1907 con la aparición de El Cuento Semanal y se prolonga hasta mediada la centuria, se observa el empleo para algunas de ellas de la denominación revista literaria. Estas colecciones de narrativa, poesía y teatro, aunque en periodicidad, distribución comercial y formato son publicaciones similares a lo que se considera una revista, constituyen en realidad una forma especial de edición y distribución del libro que se ha caracterizado por su eficacia comercial, al menos hasta la introducción de las nuevas tecnologías de la información y la comunicación. En los años treinta la denominación revista literaria también se empleó para magazines destinados al entretenimiento familiar, como la mallorquina Brisas (1934-36), creada para la promoción del turismo en la isla, o el semanario madrileño Cartel (1935), dirigido especialmente a público femenino. El empleo del adjetivo literaria parece asociado en estos y otros casos semejantes al interés por destacar, sobre los elementos gráficos del impreso, la colaboración periodística de redactores y colaboradores. La prensa española de esta etapa de esplendor de nuestra cultura alcanzó una extraordinaria calidad y una gran altura intelectual. Al margen de lo que en la actualidad se denomina periodismo literario ${ }^{4}$, la literatura tuvo una habitual e importante presencia en diarios de información general y publicaciones de toda índole: semanarios de información general, revistas culturales, magazines, revistas institucionales, estudiantiles, perió-

\footnotetext{
4 No podemos entrar en la controversia sobre la existencia o no de un periodismo literario o, como algunos quieren también, de una literatura periodística. Consideramos que periodismo y literatura constituyen ámbitos de actuación diferenciados por la función que desempeña cada uno en el sistema social. La existencia de componentes, instrumentos, normas, etc. compartidos puede explicar las estrechas y productivas relaciones intersistémicas entre estos ámbitos de actuación, pero no debería llevarnos a su plena identificación o integración. Por lo general, las intervenciones en esta controversia se fundamentan en la posibilidad de observar en los textos ciertas propiedades que consideran literarias. Nosotros, desde la perspectiva que adoptamos, consideramos que los textos no son literarios en sí mismos, sino que son los agentes que operan en el sistema literario quienes los consideran de este modo al atribuirles las características, estructuras, valores, etc. que califican como fenómenos literarios. Quiere esto decir que hoy, en función del sistema de presuposiciones bajo el que actuamos, podríamos considerar como literario un texto que en su momento fue producido para cumplir una función informativa o para expresar una opinión sobre un asunto de interés general; del mismo modo sería posible que textos que en su día se consideraron literarios no fuesen aceptados como tal por un lector actual.
} 
dicos vinculados a partidos, sindicatos, asociaciones, grupos sociales, etc. La importancia en ellas de la literatura y la presencia entre sus redactores y colaboradores de escritores destacados hacen de estas publicaciones periódicas una fuente documental imprescindible para el investigador de la actividad literaria de la época, pero no las convierte en revistas literarias.

Dificultad añadida es el uso indiferenciado por parte de críticos, estudiosos e investigadores de los adjetivos cultural y literaria para referirse a determinadas publicaciones periódicas (v. gr. Revista de Occidente). César Antonio Molina, aunque se plantea la distinción de los conceptos de prensa cultural y prensa literaria y propone incluso la correspondiente definición de cada uno ${ }^{5}$, admite que utiliza indistintamente los términos revista cultural y revista literaria «cuando en la primera de ambas hay muchos elementos (aunque no son todos) de la segunda» (1990: 21-22). Rafael Osuna, por su parte, traza las líneas generales que deben servir para la delimitación de ambos conjuntos (2004: 26):

Aunque la revista literaria pura existe en abundancia, es asimismo muy frecuente encontrarnos frente a revistas multidisciplinares que algunos gustarían llamar culturales, aunque la raya entre revista literaria y revista cultural es muy borrosa. En líneas generales, sin embargo, puede afirmarse que la revista cultural cultiva muchas preocupaciones, y entre ellas las literarias, mientras que la revista literaria tiene a esta como centro, pero también puede cultivar las restantes subsidiariamente.

La especificidad de la revista literaria como medio de comunicación social radica en constituir una acción comunicativa en el marco del sistema literario. Por lo general, las revistas de este conjunto —en el que las hay, por supuesto, consagradas por entero a la creación y/o especializadas en alguno de los géneros- integran en la estructura global de sus unidades discursivas textos literarios y no literarios ${ }^{6}$ mediante los cuales los individuos y grupos desempeñaron

5 Define la prensa cultural como «aquella que favorece el cultivo de las facultades intelectuales del hombre desde sus múltiples aspectos, tales como el literario, científico, filosófico, artístico, político, etc.» (1990: 21). Por prensa literaria entiende aquella «que se ocupa específicamente del desarrollo, divulgación, crítica y creación de esta parcela del conocimiento humano» (1990: 13).

6 Gebhard Rusch considera incompleto el sistema de la literatura descrito por la Teoría Empírica de la Literatura al limitarse a las acciones realizadas en torno a textos considerados literarios y no incluir textos que, aun sin seguir las convenciones estética y de polivalencia, son relevantes para la actividad literaria, como todos los que cumplen una función práctica (Rusch, 1994: 148). Esta diferenciación fue desarrollada posteriormente en el seno de la Teoría Empírica de la Literatura por Achim Barsch (Maldonado Alemán, 1999: 48). 
uno o varios de los papeles de actuación posibles en el sistema literario (productor, mediador, receptor o transformador de textos literarios), y algunas, incluso, textos relativos a otros ámbitos de actuación social (cultura, arte, política, etc.). No son un conjunto independiente y autónomo, sino parte del sistema hemerográfico que mantiene con otros conjuntos integrados en éste relaciones de interdependencia e intertextualidad. En los imaginarios espacios de intersección que estas relaciones de interacción trazan sobre otros conjuntos del sistema, encontramos revistas concebidas para la intervención, preferentemente, en otros ámbitos de actuación social: cultura, arte, política, educación, etc.

\section{DESCRIPCIÓN Y ANÁLISIS DE LA REVISTA LITERARIA}

La descripción y análisis de una revista literaria debe partir del hecho de que forma parte de un conjunto del sistema hemerográfico que está integrado a su vez en el sistema de medios de comunicación social en un momento determinado del devenir histórico de una sociedad. Los individuos y grupos editores de revistas literarias establecen y mantienen con ellas relaciones de interacción comunicativa con otros individuos y grupos editores de revistas en el marco de este sistema. El conjunto de las revistas en que se manifiesta esta interacción, diacrónica o sincrónicamente, constituye un módulo ${ }^{7}$. Entendemos este concepto como conjunto de referencias y modelos textuales, elemento pues del sistema de presuposiciones que orienta la actividad pública de los editores, y no como una noción instrumental para acotar un conjunto de publicaciones en el continuum hemerográfico siguiendo diferentes criterios clasificatorios. Las revistas literarias no surgen en el vacío. Los escritores que las fundan y se congregan como editores, redactores y colaboradores en torno a su publicación comparten referentes y modelos, unos intereses y expectativas (darse a conocer como escritores en los círculos literarios, promocionar su obra literaria, las ediciones de una colección editorial aneja, defender unas ideas estéticas, etc.) que pueden coincidir o discordar con los de otros grupos editores, con los que mantendrán posiblemente por ello relaciones de colaboración e intercambio o de confrontación.

Una de las primeras tareas que debe realizar el investigador de la revista literaria es determinar la composición y organigrama de la redacción. Esto lo llevará inmediatamente a indagar en la génesis, evolución y disolución

7 Rafael Osuna define este concepto como «macroestructura en que se expresa la intertextualidad de un grupo de revistas» (1998: 101) y, en otro momento, como conjunto de series que poseen elementos comunes (1998: 104). 
del grupo editor (director, secretario, redactor-jefe, redactores comprometidos con su edición) para conocer el sistema de presuposiciones compartido por sus componentes; la actividad que desarrolló el grupo en torno a la producción, difusión y recepción de revistas literarias (lo que debe incluir información sobre la tirada, forma de distribución y fuentes de financiación); su relación con los colaboradores externos de la revista y con otros grupos literarios y culturales; la interacción, por último, con el público destinatario de su actuación pública mediante otras actividades, como la edición de colecciones editoriales anejas a la revista, actos de presentación de libros, recitales, representaciones teatrales, programas radiofónicos, proyecciones fílmicas, banquetes, homenajes y otros actos sociales. Todo esto, que el investigador debe relacionar con la actividad literaria, artística y cultural del momento, conlleva la consulta de archivos documentales, fondos hemerográficos, epistolarios, autobiografías, memorias, libros de semblanzas y de recuerdos, etc. Este trabajo de documentación proporcionará sin duda valiosa información para la localización de los imprescindibles originales.

La descripción de la revista debe tener como referente cada uno de los números que componen la serie y todos sus componentes: el soporte material y la totalidad de los textos. Un rasgo distintivo de la revista literaria frente a otras publicaciones de carácter literario, como el libro o las colecciones editoriales seriadas, es la importancia que tiene su dimensión física, el impreso o soporte material (Osuna, 1998: 7-11). Mientras que en el libro tradicional o en la colección seriada la edición de una obra literaria se realiza en un soporte circunstancial que puede variar de una edición a otra y cuya función es preservar la inmutabilidad del texto en tanto que base de comunicado, en la revista literaria el soporte material (formato, papel, tintas, diseño gráfico) es invariable, único en el tiempo, inseparable del componente lingüístico con el que forma la unidad discursiva y, como este, sujeto a las normas estéticas del sistema de presuposiciones bajo el que operan en el sistema literario individuos y grupos. Se debe tener en cuenta, no obstante, que los recursos económicos y técnicos a disposición del grupo editor pueden condicionar o determinar en última instancia su producción.

El título, que identifica a la revista como entidad, es el elemento representativo, el más cuidado por los editores, su imagen. Sintetiza, como componente gráfico y lingüístico, sus valores estéticos, la posición de la revista en el sistema y su inserción en un módulo hemerográfico. Baste recordar, por poner un ejemplo, el conjunto de revistas literarias que en los años veinte y treinta llevaron como título una referencia geográfica: Mediodía, Meseta, Meridiano, Sudeste, Noreste, Isla, entre otras. 
Aunque la revista quede identificada como entidad, se debe tener en cuenta que constituye en realidad una serie de números en secuencia temporal y causal, esto es, un conjunto de unidades discursivas producidas, transmitidas y recibidas secuencialmente en diferentes y sucesivos actos de comunicación. Frente a lo que se pudiese pensar en un primer momento, la serie, y por lo tanto la revista, no equivale a la suma de cada una de las unidades que la componen ni puede ni debe ser considerada instrumento - en el sentido de texto- empleado para la realización de una acción comunicativa. La serie es la representación cognitiva tanto del conjunto como del proceso seguido en la secuenciación de unidades e incluye, junto a la representación cognitiva de cada unidad, las regularidades observadas a lo largo de la cadena y los cambios y transformaciones operados en el soporte material, en la estructuración de las unidades y, ascendiendo en la escala de abstracción, en el sistema de presuposiciones bajo el que actúan los individuos y grupos en cada una de las acciones comunicativas. Esta representación cognitiva, que integra el modelo textual empleado para la producción y recepción de cada unidad, es elemento fundamental de la estrategia de comunicación y se actualiza con la salida de cada nuevo número. En este sentido, la producción y recepción de cada unidad está condicionada, entre otros factores, por las unidades previas a partir de las cuales se construye la serie, al tiempo que condiciona la producción y recepción de las unidades siguientes.

Cada número de una revista es, pues, una acción comunicativa independiente como tal, pero no un acontecimiento aislado. Se produce en una determinada situación comunicativa con sus propias circunstancias y en concurrencia con otros agentes comunicativos, con otras acciones comunicativas, etc., de tal manera que factores externos a los sistemas hemerográfico y literario - de carácter político, económico, por ejemplo- o internos - como la publicación de una obra literaria, la recepción de los números anteriores, la aparición de una nueva revista, la publicación de nuevos números de otras publicaciones, etc. - pueden alterar las condiciones de producción y el sistema de presuposiciones del que forma parte la estrategia de comunicación del individuo o grupo editor. Quiere todo esto decir que el número, en su integridad y como unidad discursiva, es un elemento que debe ser analizado de modo independiente en el conjunto de la serie, en relación con el módulo en que la revista se integra y teniendo en cuenta el contexto de actuación de los editores. Con este análisis sincrónico se atiende a su historicidad y a las relaciones de intertextualidad con números de otras series e incluso con otros números de la misma. Se debe tener en cuenta al respecto, no obstante, que la periodicidad de una revista influye 
en el grado de dependencia de cada número. Los números de una revista de periodicidad irregular y salidas muy espaciadas son más independientes entre sí y más receptivos a los estímulos procedentes de fuentes externas que los de una revista de mayor periodicidad y con una bien definida estructuración y orientación de sus secciones y contenidos. Además, algunas revistas se crean y publican con unos objetivos precisos, siguiendo una orientación editorial muy definida y sobre un sólido proyecto empresarial o institucional que proporciona la estabilidad y la regularidad de que carecen por lo general las revistas literarias, publicadas normalmente por individuos o grupos sin estructura empresarial ni vínculos, en cuanto productores y editores, con instituciones sociales, políticas y culturales. En estos casos, la incidencia de factores externos en la composición de cada uno de los números es menor o casi nula.

La descripción de cada número debe atender a la estructuración de la unidad, al conjunto de los textos que la componen (lo que incluye la publicidad, cuyo análisis aportará información sobre las fuentes de financiación y el público a que se dirige) y a la cotextualidad generada por la disposición de los textos en el espacio simbólico ${ }^{8}$. Los textos que componen la revista no se deben tomar como textos aislados, independientes, sino como elementos de una unidad que condiciona, como cotexto general que integra otros cotextos, la representación cognitiva o significado que los receptores les asignan. Se debe tener en cuenta para el análisis, por tanto, la totalidad de los textos que componen la revista, independientemente de su consideración de textos estéticos o no estéticos, artísticos o literarios. El examen del conjunto de los textos proporcionará información acerca del sistema de presuposiciones que gobierna la actuación del individuo o grupo editor, especialmente en lo concerniente a formas textuales, temas y elementos estilísticos de uso preferente; valores estéticos e ideológicos compartidos, discutidos y rechazados por el grupo; modelos literarios y artísticos seguidos; conocimiento y valoración de la tradición literaria y artística; influencias recibidas por los individuos o grupos procedentes de otros individuos y grupos o de otros sistemas literarios y artísticos, etc. Las páginas de las revistas literarias son por ello un campo privilegiado para la observación de fenómenos de transferencia, canonización e interferencia (Iglesias Santos, 1994: 331-339).

\footnotetext{
8 Por espacio simbólico se entiende la significación atribuida por productores y receptores, según determinadas convenciones, al lugar del impreso ocupado por uno o un conjunto de textos con respecto a la página, la sección, el número, la serie o incluso, con respecto al lugar ocupado por otro texto o conjunto de textos (Osuna,1998: 11-16).
} 
Además, editoriales, notas, reseñas, críticas, recensiones y homenajes son importante fuente de información sobre las actividades realizadas por autores y grupos literarios y culturales, las relaciones mantenidas entre ellos y sobre las propias revistas literarias.

La elaboración de índices con toda la información procedente de la descripción y análisis de cada número es una de las tareas más comunes en el ámbito de los estudios hemerográficos. Constituyen una fuente documental básica para la actividad investigadora, pueden suplir provisionalmente el manejo directo de originales o copias y facilitan un primer y rápido acceso a información sobre autores y textos publicados. Las ediciones facsimilares de revistas literarias suelen incluir como anexos los sumarios de cada número y un índice onomástico de autores. La mayoría de estos índices, sin embargo, tienen una funcionalidad limitada, pues no suelen proporcionar información sobre el género de la colaboración y, cuando lo hacen, se limitan a distinguir por lo general el verso de la prosa. Más útiles que estos índices son, sin duda, las bases de datos digitales. Ponen en manos del investigador una herramienta capaz de proporcionar con rapidez documentación necesaria para su tarea. Además, sus registros pueden ser integrados posteriormente en otras bases de datos mayores con las que se podrían generar índices múltiples del conjunto de las revistas que componen un módulo hemerográfico e incluso de las de toda una época. Desde luego, la funcionalidad de estas bases de datos no reside solo en el soporte digital. Para que sean realmente útiles cada registro debe proporcionar toda la información relativa a cada uno de los textos que componen la revista: autoría, título, título de los poemas que componen la colaboración o primeros versos, nombre de la revista, número, fecha, lugar de edición y género de la colaboración según las categorías establecidas por las convenciones más extendidas sobre géneros literarios y hemerográficos; en el caso de las críticas, reseñas y recensiones, además, autoría del producto cultural a que remite (libro, periódico, revista, obra de teatro, film, etc.) y título de este.

\section{REVISTAS LITERARIAS Y NUEVAS TECNOLOGÍAS}

Desde hace unos años se asiste a un salto cualitativo en la conservación y difusión de fondos hemerográficos mediante la publicación en Internet de copias digitalizadas. Esto pone al alcance del investigador, y de cualquier lector interesado, copias de revistas literarias que hasta hace poco eran de difícil acceso. Téngase en cuenta que la mayor parte de las revistas literarias tuvieron tiradas reducidas y que de algunas de ellas solo se han conservado 
contados originales, dispersos en muchos casos, además, en archivos y bibliotecas. La labor que se ha desarrollado en España hasta este momento adolece, sin embargo, de falta de coordinación. Cada institución digitaliza y publica sus propios fondos hemerográficos y esto tiene como consecuencia la dispersión del conjunto en Internet y la publicación incluso de series y conjuntos fragmentarios. Un catálogo exhaustivo de las revistas literarias que se han publicado a lo largo del tiempo, elaborado a partir de la descripción de originales de series completas y con enlaces a los servidores web que contienen copias digitalizadas de cada una de ellas, sería el instrumento perfecto para la búsqueda y el complemento imprescindible de las bases de datos.

Todo lo que se ha expuesto en el apartado anterior ha sido escrito con el referente de la revista literaria impresa, pero es válido también para aquellas revistas que utilizan el soporte digital (archivo PDF o en cualquier otro formato alojado en servidor web, en $\mathrm{CD}$, etc.) por su menor coste de producción y su facilidad de difusión. Mantienen las convenciones del producto cultural asentadas en el sistema literario desde los inicios del siglo XX y solo se diferencian de la revista literaria impresa en la posibilidad de incluir hipervínculos. De hecho, algunas revistas literarias combinan actualmente la edición digital con una pequeña tirada impresa que en nada se diferencian. Como este artículo, pueden leerse del mismo modo sobre el papel o en una pantalla de cristal líquido.

Los cambios que están introduciendo las nuevas tecnologías de la información y la comunicación son, sin embargo, más profundos. Del mismo modo que los avances técnicos que comenzaron a introducirse al finalizar el siglo XIX en los procesos de diseño, composición e impresión hicieron posible que la revista literaria adquiriese su moderna fisonomía y propiciaron incluso la experimentación formal en la poesía de aquellos años, las nuevas tecnologías de la información y la comunicación afectan a la actividad literaria en general (a la creación y a la recepción de las obras, a la interacción del escritor con sus lectores, a las formas de producción y comercialización, etc.) y están transformando la naturaleza de la revista literaria, concebida ya como un espacio hipermedia en el que textos, fotografías, vídeos, sonidos e hipervínculos se entrelazan formando un continuo de información no secuencial abierto a la interacción con el público. Frente al espacio finito de la página, su relación secuencial con el resto de las páginas del número y su inmutable composición, el espacio hipermedia se ofrece al nuevo lector con la labilidad de un laberinto borgiano. Impone la fragmentación y no permite el contacto físico con la totalidad del objeto. Obviamente, estas característi- 
cas de la revista literaria digital demandan una nueva metodología y exigen nuevos planteamientos en lo relativo a su conservación y catalogación.

\section{CONCLUSIÓN}

Es evidente que el conjunto de las revistas literarias de una época constituye un corpus imprescindible para la observación de todo tipo de acciones, procesos y fenómenos literarios y, por tanto, para la descripción y explicación de la organización y estructura del sistema. Su singularidad como medio de comunicación demanda del investigador un tratamiento diferenciado y una metodología propia. La colaboración literaria de escritores en periódicos de información general, magazines y revistas de cualquier otro género - en los que el escritor no es más que un colaborador externo que no interviene, en cuanto autor literario, en su gestación y producción- está supeditada a los propósitos e intereses de la empresa editorial, situados por lo general en ámbitos de actuación social distintos del literario. En estos casos el investigador distingue en el conjunto los textos que tienen interés para su actividad investigadora y prescinde del resto y aun de la propia publicación, que queda como simple continente y dato referencial. En cambio, la revista literaria — sobre cuya gestación, producción y difusión los autores literarios suelen ejercer un dominio pleno- es un producto literario en sí que debe ser examinado, descrito y analizado como tal, atendiendo tanto a su materialidad como a la totalidad de los textos que lo componen. Las nuevas tecnologías de la información y la comunicación han allanado sin duda la tarea de los investigadores, pero traen consigo cambios profundos que exigirán un nuevo enfoque para los estudios hemerográficos.

\section{REFERENCIAS BIBLIOGRÁFICAS}

CHICO RICO, F. (1995). «Introducción a la Ciencia Empírica de la Literatura». Teoría/Crítica 2, 11-34.

DÍEZ DE REVENGA, F. J. (2005). Poetas y narradores. La narrativa breve en las revistas de vanguardia en España (1918-1936). Madrid: Devenir.

EVEN-ZOHAR, I. (1999). «Factores y dependencias en la cultura. Una revisión de la Teoría de los Polisistemas». En Teoría de los Polisistemas, Montserrat Iglesias Santos (comp.), 23-52. Madrid: Arco / Libros.

GRACIA, J. (2004). «El bullicio democrático». Quimera 250, 15-17. 
IGLESIAS SANTOS, M. (1994). «El sistema literario: Teoría Empírica y Teoría de los Polisistemas». En Avances en Teoría de la Literatura, Darío Villanueva (comp.), 309-356. Santiago de Compostela: Universidad de Santiago de Compostela.

- (1999). «La teoría de los polisistemas como desafío a los estudios literarios». En Teoría de los Polisistemas, Montserrat Iglesias Santos (comp.), 9-20. Madrid: Arco / Libros.

MALDONADO ALEMÁN, M. (1999). «El pensamiento sistémico en la teoría literaria alemana I». Revista de Filología Alemana 7, 15-60.

- (2006). «La historiografía literaria. Una aproximación sistémica». Revista de Filología Alemana 14, 9-40.

MOLINA, C. A. (1990). Medio siglo de prensa literaria española. Madrid: Endymión.

OSUNA, R. (1998). Tiempo, materia y texto. Una reflexión sobre la revista literaria. Kassel: Edition Reichenberger.

- (2004). Las revistas literarias. Un estudio introductorio. Cádiz: Servicio de Publicaciones de la Universidad de Cádiz.

RAMOS ORTEGA, M. J. (2005). «Introducción». Revistas literarias españolas del siglo XX (1919-1975). Volumen I (1919-1939), Manuel J. Ramos Ortega (ed.), 175-178. Madrid: Ollero y Ramos.

RÓDENAS DE MOYA, D. (2004). «La prensa cultural en la Edad de Plata». Quimera 250, 18-22.

RUSCH, G. (1994). «Teorías sistémicas en la Historiografía Literaria alemana». Teoría/Crítica 1, 137-172.

SCHMIDT, S. J. (1991). Fundamentos de la ciencia empírica de la literatura. Madrid: Taurus.

- (1995a). «La Ciencia Empírica de la Literatura: Un nuevo paradigma». Teoría/Crítica 2, 87-106.

- (1995b). «Escribir historias de la literatura. Algunas observaciones desde un punto de vista constructivista». Teoría/Crítica 2, 245-269.

Recibido el 3 de mayo de 2013.

Aceptado el 19 de septiembre de 2013. 
